\title{
Suplementação de vitamina D na prevenção de quedas e fraturas em idosos: uma revisão baseada na evidência
}

Anabela Barreto Silva, ${ }^{1}$ Maria João Barbosa²

\section{RESUMO}

Introdução: As quedas e fraturas constituem uma importante causa de hospitalização e mortalidade em indivíduos idosos, contribuindo de forma significativa para a sua perda de autonomia. O papel da vitamina D na melhoria dos distúrbios músculo-esqueléticos em doentes idosos na prevenção de quedas e fraturas é ainda controverso.

Objetivos: Determinar a evidência do efeito da suplementação da vitamina $D$ na prevenção de quedas e fraturas em idosos.

Fonte de dados: MEDLINE, The Cochrane Library, NGC, NICE, DARE, TRIP Database, USPSTF, AGS e DGS.

Métodos: Pesquisa de estudos publicados nos últimos 10 anos (de 01 de fevereiro de 2009 a 28 de fevereiro de 2019), escritos em Português e Inglês, utilizando os termos MeSH Vitamin D, Accidental Falls e Fractures, Bone. Para avaliação da qualidade dos estudos e força de recomendação foi utilizada a escala Strength of Recommendation Taxonomy, da American Family Physician (SORT).

Resultados: Da pesquisa inicial resultaram 514 artigos, tendo sido selecionados 15 por cumprirem os critérios de inclusão definidos: seis guidelines/normas de orientação clínica (NOC), oito revisões sistemáticas (RS) e meta-análises (MA) e um ensaio clínico aleatorizado (ECA). A maioria das guidelines e NOC defende a suplementação de vitamina D na prevenção de quedas e fraturas em idosos. Contudo, a análise das RS e MA mostram resultados discordantes ao demonstrar que a suplementação de vitamina $D$ não tem efeitos nas quedas e fraturas em idosos, nomeadamente não institucionalizados.

Conclusões: Em idosos não institucionalizados e sem défice de 25(OH)D não há benefício da suplementação de vitamina na prevenção de quedas e fraturas (SORT B). Idosos institucionalizados e com défice de $25(\mathrm{OH}) \mathrm{D}$ devem ser suplementados com vitamina $D$ isoladamente ou combinada com cálcio (SORT B). A suplementação de doses elevadas de vitamina $D$ e de forma intermitente pode ter efeitos deletérios (SORT B).

Palavras-chave: Vitamina D; Quedas; Fraturas; Idosos.

\section{INTRODUÇÃO}

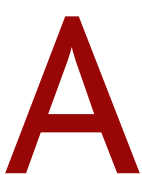
s quedas e lesões subsequentes são um contributo major para a perda de capacidades associadas ao envelhecimento, constituindo uma importante causa de hospitalização e mortalidade em indivíduos idosos. Aproximadamente $28-35 \%$ das pessoas com idade igual ou superior a 65 anos têm pelo menos um episódio de queda a cada ano. Uma em cada cinco quedas necessita de cuidados

1. Médica Interna de Medicina Geral e Familiar. USF Gualtar. 2. Médica Assistente de Medicina Geral e Familiar. USF Gualtar. médicos e uma em cada vinte termina em fratura. ${ }^{1}$ As quedas, além de originarem uma considerável perda de autonomia e de qualidade de vida entre os idosos, podem ainda ter repercussões nos seus cuidadores, frequentemente os familiares, que passam a ter novas exigências em função da recuperação ou adaptação após a queda. Por esta razão, é importante desenvolver medidas preventivas exequíveis e efetivas, de modo a reduzir o número de quedas e fraturas nesta população. Abordagens multifatoriais, como exercício físico, terapia ocupacional, ajustes terapêuticos e modificação do ambiente, podem ser úteis. Contudo, estas podem ser 
dispendiosas, difíceis de implementar e muito dependentes da adesão do indivíduo. A deficiência em vitamina $\mathrm{D}$, comum nos idosos, ${ }^{2-3}$ precipita ou agrava a osteopenia e osteoporose, interfere no desenvolvimento muscular e, consequentemente, aumenta o risco de quedas e fraturas. Vários estudos têm investigado o papel da vitamina D na melhoria dos distúrbios músculo-esqueléticos com resultados conflituosos. Pretende-se, com esta revisão, determinar a evidência do efeito da suplementação da vitamina $D$ na prevenção de quedas e fraturas em idosos.

\section{MÉTODOS}

Foi feita uma pesquisa da literatura publicada nos últimos 10 anos (de 01 de fevereiro de 2009 a 28 de fevereiro de 2019), nas línguas inglesa e portuguesa, com as seguintes combinações dos termos MeSH Vitamin D, Accidental Falls e Fractures, Bone. As fontes de informação consultadas foram: National Guideline Clearinghouse (NGC), National Institute for Health and Care Excellence Guidelines Finder (NICE), TRIP Database, The Cochrane Library, Database of Abstracts of Reviews of Effectiveness (DARE), MEDLINE/PubMed, United States Preventive Services Task Force (USPSTF), American Geriatric Society (AGS) e Direção-Geral da Saúde (DGS). Para atribuição dos níveis de evidência (NE) e forças de recomendação (FR) foi utilizada a escala Strength of Recommendation Taxonomy (SORT), da American Family Physician. No que diz respeito aos critérios de elegibilidade foi utilizado o modelo PICOS (Population, Intervention, Comparison, Outcome, Studies), de forma a definir as variáveis a avaliar. Assim, foram consideradas para população em estudo todas as pessoas do género feminino ou masculino com idade $\geq 65$ anos, não institucionalizadas e institucionalizadas. Estudos em que a média de idades dos participantes seria $\geq 65$ anos foram incluídos. Não foi definido um período mínimo de follow-up para a inclusão dos estudos. Foram excluídos todos os artigos que envolveram participantes com osteoporose diagnosticada ou sob tratamento de vitamina D. A intervenção consistiu na suplementação de vitamina $D$. Estudos que envolveram a vitamina D combinada com outras intervenções, exceto o cálcio, foram excluídos. O grupo comparador abrangeu participantes que não foram suplementados com vitamina $\mathrm{D}$. Ocorrência de quedas e fraturas foram os outcomes avaliados. Foram incluídos guidelines, normas de orientação clínica (NOC), revisões sistemáticas (RS), meta-análises (MA) e estudos aleatorizados (ECA). Excluíram-se outro tipo de artigos, nomeadamente os de custo-efetividade. Assim, foram aplicados os filtros clinical trial, review e guidan$c e$. Foram também excluídos artigos de opinião, editoriais, letters e notícias, e estudos não aleatorizados ou realizados em animais. Estudos duplicados ou já incluídos nas RS ou MA selecionadas foram também excluídos.

\section{RESULTADOS}

Da pesquisa inicial resultaram 514 artigos, dos quais restaram 479 após a eliminação dos duplicados. Após leitura do título e do abstractresultaram 29 artigos, tendo sido selecionados 15 artigos para integrar a revisão por cumprirem os critérios de inclusão definidos. O fluxograma de seleção dos artigos está representado na Figura 1. No final obtiveram-se seis guidelines, oito RS/MA e um ECA. Uma vez que o tipo de artigos incluídos, desenhos de estudos, participantes e medidas de outcomes variam significativamente, a apresentação dos resultados será feita de forma qualitativa em vez de MA. As Tabelas 1, 2, 3 e 4 resumem as características dos estudos selecionados para esta revisão.

\section{Guidelines e normas de orientação clínica}

Atualmente as guidelines e NOC apresentam recomendações contraditórias relativamente à suplementação de vitamina D na prevenção de quedas e fraturas em idosos. As últimas recomendações da USPTF $(2018)^{4}$ vêm contrariar a recomendação feita em 2012, assumindo uma posição contra a suplementação de vitamina D na prevenção de quedas em adultos com $\geq 65$ anos não institucionalizados, que não possuem défice de vitamina D ou osteoporose. Esta orientação foi baseada na RS de Guirguis-Blake e colaboradores, ${ }^{5}$ publicada em 2018, a qual consiste numa atualização à RS de 2010, com notáveis diferenças. Isto é, ao contrário da anterior, desta revisão foram excluídos estudos que recrutaram participantes com défice ou níveis insuficientes de vitamina D. Assim, trata-se de uma recomendação SORT B, uma vez que é baseada em revisões de ECA de qualidade heterogénea e cujos resultados dos poucos ensaios que foram incluídos são pouco 


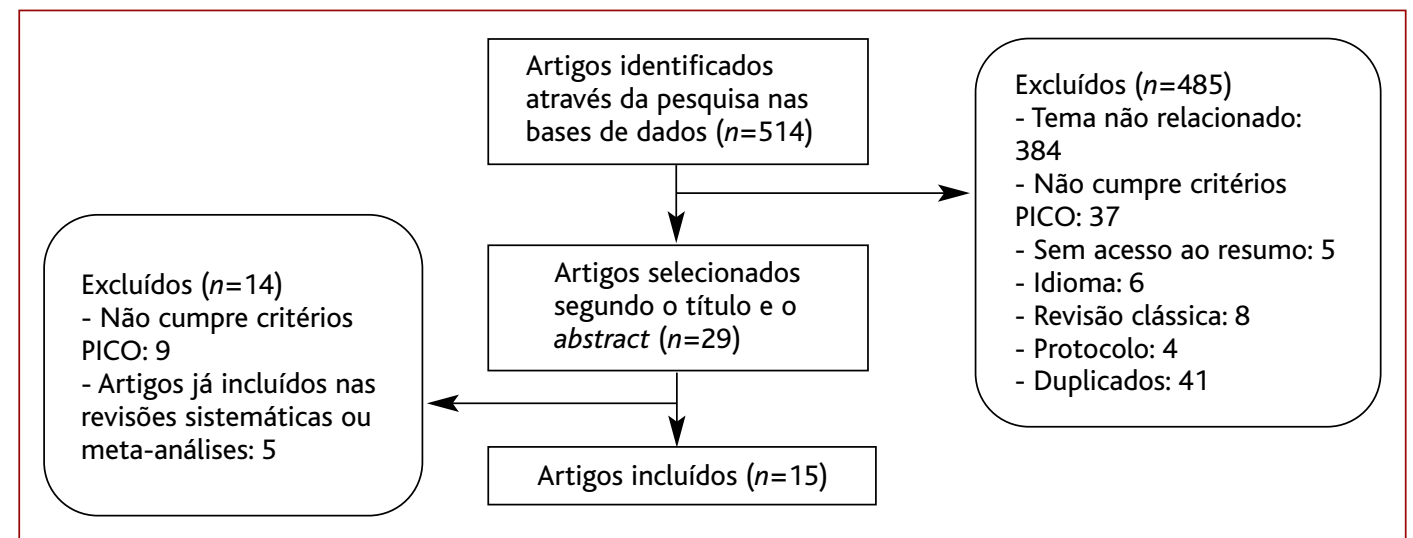

Figura 1. Fluxograma de seleção dos artigos.

consistentes. Contrariamente, as orientações da $\mathrm{Na}$ tional Osteoporosis Society (2018) ${ }^{6}$ são a favor da suplementação de vitamina D na dose de $400 \mathrm{IU}$ a indivíduos $c o m \geq 65$ anos não expostos frequentemente ao sol. Por sua vez, os consensos da AGS (2014) ${ }^{7}$ admitem que a suplementação de vitamina $D$ deve ser feita a idosos institucionalizados e não institucionalizados, na dose mínima de 1.000 UI e em associação com o cálcio. Em 2010, a International Osteoporosis Foundation (IOF) lançou uma declaração, referindo que para que sejam atingidos níveis séricos de 25- hidroxivitamina $\mathrm{D}$ $(25(\mathrm{OH}) \mathrm{D}) \mathrm{de}$ $30 \mathrm{ng} / \mathrm{ml}$ são necessárias doses diárias entre 800-1.000 IU de vitamina D. Considera ainda que não existe evidência que permita afirmar que doses superiores possam ser eficazes na prevenção de quedas e fraturas em idosos. Do mesmo modo, em 2008 a DGS 9 lançou uma circular informativa com a indicação para suplementar idosos com vitamina D e cálcio, admitindo tratar-se de uma população de risco para fratura. Estas recomendações são baseadas no consenso da opinião de autores e, por essa razão, são classificadas como SORT C. A Endocrine Society ${ }^{10}$ faz uma

TABELA 1. Resumo de guidelines e normas de orientação clínica

\begin{tabular}{l|l} 
Referência & \multicolumn{1}{c}{ Recomendação } \\
\hline $\begin{array}{l}\text { United States Preventive } \\
\text { Task Force (2018) }\end{array}$ & $\begin{array}{l}\text { Não é recomendada a suplementação de vitamina D para a prevenção de quedas em adultos com } \\
\geq 65 \text { anos não institucionalizados. (SORT B) }\end{array}$ \\
\hline $\begin{array}{l}\text { National Osteoporosis } \\
\text { Society (2018) }\end{array}$ & $\begin{array}{l}\text { Adultos com } \geq 65 \text { anos que não são expostos ao sol devem receber diariamente } 400 \text { IU de vitamina } \\
\text { D. (SORT C) }\end{array}$ \\
\hline $\begin{array}{l}\text { Consensos American } \\
\text { Geriatrics Society (2014) }\end{array}$ & $\begin{array}{l}\text { Está recomendada a suplementação com vitamina D de pelo menos 1000 IU e suplementação de } \\
\text { cálcio: } \\
\text { - Adultos com idade } \geq 65 \text { anos não institucionalizados, de forma a reduzir o risco de quedas e } \\
\text { fraturas. (SORT C) } \\
\text { - Idosos institucionalizados, de forma a reduzir o risco de quedas e fraturas. (SORT C) } \\
\text { Não existe evidência para apoiar a suplementação de vitamina D sem cálcio a idosos } \\
\text { institucionalizados e não institucionalizados. (SORT C) }\end{array}$ \\
\hline $\begin{array}{l}\text { Endocrine Society (2011) } \\
\text { Recomendam a suplementação de 800 IU/d de vitamina D a adultos com idade } \geq 65 \text { anos para a } \\
\text { prevenção de quedas e fraturas. (SORT A) }\end{array}$ \\
\hline $\begin{array}{l}\text { International Osteoporisis } \\
\text { Foundation (2010) }\end{array}$ & $\begin{array}{l}\text { Indivíduos idosos requerem 800-1000 IU/d de vitamina D. Consideram a inexistência de evidência } \\
\text { para recomendar doses mais altas na prevenção de quedas e fraturas. (SORT C) }\end{array}$ \\
\hline $\begin{array}{l}\text { Direção-Geral da Saúde } \\
\text { (2008) }\end{array}$ & $\begin{array}{l}\text { Adultos com } \geq 65 \text { anos têm indicação para realizar suplementação com cálcio e vitamina D, de } \\
\text { forma a diminuir o risco de fratura. (SORT C) }\end{array}$ \\
\hline
\end{tabular}




\begin{tabular}{|c|c|c|c|}
\hline Referência & $\begin{array}{l}\text { Estudos } \\
\text { incluídos }\end{array}$ & Resultados & NE \\
\hline $\begin{array}{l}\text { Guirguis- } \\
\text {-Blake, et al. } \\
(2018)^{11}\end{array}$ & $\begin{array}{c}7 \text { ECAS } \\
(n=7.531)\end{array}$ & 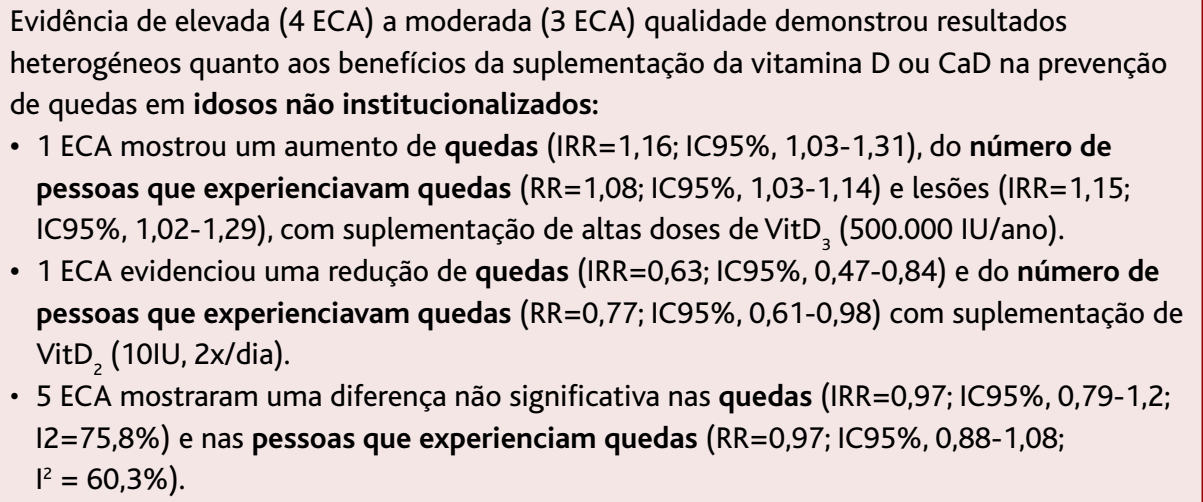 & 1 \\
\hline $\begin{array}{l}\text { Cameron, et } \\
\text { al. }(2018)^{12}\end{array}$ & $\begin{array}{c}4 \text { ECA } \\
(n=4.512)\end{array}$ & $\begin{array}{l}\text { Em idosos institucionalizados após a suplementação com } \mathrm{VitD}_{2} \text { ou } \mathrm{CaD}\left(\mathrm{VitD}_{3} \text { e VitD }{ }_{2}\right) \text { : } \\
\text { - Verificou-se uma redução estatisticamente significativa na taxa de quedas ( } \mathrm{RaR}=0,72 \text {; } \\
\left.\mathrm{IC} 95 \%, 0,55-0,95 ; \mathrm{I}^{2}=62 \%\right) \text {, mas não no risco de queda ( } \mathrm{RR}=0,92 ; \mathrm{IC} 95 \%, 0,76-1,12 ; \\
\left.\mathrm{I}^{2}=42 \%\right) \text {. }\end{array}$ & 2 \\
\hline $\begin{array}{l}\text { Wu, et al. } \\
(2017)^{13}\end{array}$ & $\begin{array}{c}26 \text { ECA } \\
(n=16.540)\end{array}$ & $\begin{array}{l}\text { Em idosos institucionalizados e não institucionalizados não foi encontrada diferença } \\
\text { estatisticamente significativa na redução do risco de queda em: } \\
\text { - } 6 \text { ECA com a suplementação de } \operatorname{VitD}_{2}\left(\mathrm{OR}=0,77 ; \mathrm{IC} 95 \%, 0,58-1,03 ; I^{2}=79 \%\right) \text {. } \\
\text { - } 6 \mathrm{ECA} \text { com a suplementação de } \operatorname{VitD}_{3}\left(\mathrm{OR}=1,08 ; \mathrm{IC} 95 \%, 0,98-1,20 ; I^{2}=42 \%\right) \text {. } \\
\text { - } 15 \mathrm{ECA} \text { demonstram uma associação significativa entre a suplementação de vitamina } \mathrm{D} \\
\left.\text { combinada com cálcio e a redução do risco de queda (OR=0,87; IC95\%, } 0,80-1,94 ; I^{2}=46 \%\right) \text {. }\end{array}$ & 1 \\
\hline $\begin{array}{l}\text { Zheng, Ya } \\
\text { Ting, et al. } \\
(2015)^{14}\end{array}$ & $\begin{array}{c}9 \text { ECAS } \\
(n=22.012)\end{array}$ & $\begin{array}{l}\text { Em idosos institucionalizados e não institucionalizados suplementados com doses elevadas } \\
\text { de vitamina } D \text { e intermitentes: } \\
\left.\text { - } 3 \text { ECA mostraram um aumento de quedas ( } R R=1,02 ; \mathrm{IC} 95 \%, 0,96-1,08 ; p=0,52 ; I^{2}=58 \%\right) \text {. } \\
\text { - } 4 \text { ECA evidenciaram aumento de fraturas da anca ( } R R=1,17 ; \mathrm{IC} 95 \%, 0,97-1,41 ; p=0,11 ; \\
\left.\mathrm{I}^{2}=10 \%\right) \text {. } \\
\text { - } 5 \text { ECA demonstraram um aumento de fraturas não vertebrais ( } R R=1,06 ; \mathrm{IC} 95 \%, 0,91-1,22 ; \\
\left.P=0,48 ; I^{2}=54 \%\right) \text {. }\end{array}$ & 1 \\
\hline $\begin{array}{l}\text { Bolland, et al. } \\
(2014)^{15}\end{array}$ & $\begin{array}{c}22 \text { ECA } \\
(n=24.903)\end{array}$ & $\begin{array}{l}\text { Em idosos institucionalizados e não institucionalizados: } \\
\text { - } 22 \text { ECA não demonstraram alteração do risco de fratura total em } \geq 15 \% \text { após a } \\
\text { suplementação com vitamina } D \text { isolada ou combinada com cálcio ( } R R=0,95 ; I C 95 \% \text {, } \\
0,88-1,02 ; p=0,13 ; I^{2}=33 \% \text { ). } \\
\text { - } 12 \text { ECA não demonstraram diminuição do risco de fratura do colo do fémur } \geq 15 \% \text { após a } \\
\text { suplementação de vitamina } D \text { apenas ( } R R=1,11 ; I C 95 \%, 0,97-1,27 ; p=0,13 ; I^{2}=0 \% \text { ). } \\
\text { - } 2 \text { ECA demonstraram uma redução do risco de fratura em indivíduos institucionalizados } \\
\text { após a suplementação combinada de cálcio e vitamina } D \text {. } \\
\text { - } 7 \text { ECA não se verificou alteração do risco de fratura da anca } \geq 15 \% \text { em indivíduos não } \\
\text { institucionalizados após a suplementação combinada de cálcio e vitamina } D \text {. }\end{array}$ & 1 \\
\hline
\end{tabular}

forte recomendação para a suplementação de $800 \mathrm{IU} / \mathrm{d}$ de vitamina $\mathrm{D}$, baseando-se em evidência de elevada qualidade, pelo foi classificada como SORT A.

\section{Revisões sistemáticas e meta-análises}

Resultados mistos foram obtidos através da análise da RS de Guirguis-Blake e colaboradores (2018) ${ }^{11}$ 
TABELA 2. Resumo de revisões sistemáticas e meta-análises (continuação)

\begin{tabular}{|c|c|c|c|}
\hline Referência & $\begin{array}{l}\text { Estudos } \\
\text { incluídos }\end{array}$ & Resultados & NE \\
\hline $\begin{array}{l}\text { Gui, et al. } \\
(2013)^{16}\end{array}$ & $\begin{array}{c}11 \text { ECA } \\
(n=9.750)\end{array}$ & $\begin{array}{l}\text { Em doentes idosos não institucionalizados e sem défice cognitivo: } \\
\text { - } 6 \text { ECA demonstraram redução do risco de queda com a suplementação de cálcio com } \\
\text { vitamina } D(O R=0,789 ; \mathrm{IC} 95 \%, 0,631-0,985 ; p=0,036) \text {. } \\
\text { - } 5 \text { ECA não demonstraram redução do risco de queda com a suplementação de vitamina } D \\
\text { isoladamente }(O R=1,026 ; \mathrm{IC} 95 \%, 0,819-1,286 ; p=0,036) \text {. }\end{array}$ & 1 \\
\hline $\begin{array}{l}\text { Gillespie, et } \\
\text { al. }(2012)^{17}\end{array}$ & $\begin{array}{c}14 \mathrm{ECA} \\
(n=28.135)\end{array}$ & $\begin{array}{l}\text { Em idosos não institucionalizados não existe uma diferença estatisticamente significativa } \\
\text { com a suplementação de vitamina } D \text { isolada ou em combinação com o cálcio: } \\
\text { - } 7 \text { ECA - na taxa de quedas ( } \operatorname{RaR}=1,00 ; \mathrm{IC} 95 \%, 0,90-1,11 ; p<0,00001) \text {. } \\
\text { - } 13 \mathrm{ECA} \text { - no risco de queda (RR=0,96; IC95\%, 0,89-1,03; } p<0,00001) \text {. } \\
\text { - } 10 \mathrm{ECA} \text { - no risco de fratura (RR=0,94; IC95\%, 0,82-1,09; } p<0,00001) \text {. }\end{array}$ & 1 \\
\hline $\begin{array}{l}\text { Lai, et al. } \\
(2010)^{18}\end{array}$ & $\begin{array}{c}7 \text { ECA } \\
(n=12.739)\end{array}$ & $\begin{array}{l}\text { Em indivíduos idosos, institucionalizados e não institucionalizados: } \\
\text { Não foi demonstrada diferença estatisticamente significa no risco de fratura da anca após a } \\
\text { suplementação com vitamina D3 ou vitamina D2 ( } R R=1,13 ; \text { IC95\%, } 0,98-1,29 ; p=0,73 ; \\
\chi 2=4,44) \text {, não verificando diferença entre os ECA que utilizaram doses }<800 \mathrm{IU} / \mathrm{d} \text { em } \\
\text { aqueles com } \geq 800 \mathrm{IU} / \mathrm{d} \text {. }\end{array}$ & 2 \\
\hline
\end{tabular}

Legenda: $\mathrm{ECA}=$ Ensaio clínico aleatorizado; $\mathrm{RaR}=$ Rate ratio; $\mathrm{RR}=$ Relative risk; $\mathrm{OR}=$ Odds ratio.

TABELA 3. Resumo de ensaios clínicos aleatorizados

\begin{tabular}{|l|l|c} 
Referência & Resultados & NE \\
\hline Smith (2017) ${ }^{19}$ & Em mulheres idosas não institucionalizadas suplementadas com vitamina D: & 1 \\
& $\begin{array}{l}\text { - Não houve diminuição das quedas com baixas doses de vitD }{ }_{3^{\prime}} 400 \text { e } 800 \text { IU, uma diminuição } \\
\text { significativa com doses médias, } 1.600,2.400,3.200 \mathrm{IU}(p=0,002) \text { e houve aumento com altas doses, } \\
4.000 \text { e } 4.800 \text { IU comparativamente com o placebo }(p=0,55) .\end{array}$ &
\end{tabular}

Legenda: VitD $_{3}=$ Colecalciferol.

quanto à suplementação de vitamina $\mathrm{D}$ isoladamente ou combinada com o cálcio $(\mathrm{CaD})$ na prevenção de quedas em idosos não institucionalizados. Isto é, um ECA mostrou uma redução estatisticamente significativa do risco de queda (IRR=0,63; IC95\%, 0,47-0,84), enquanto um ECA demonstrou um aumento do risco de queda quando utilizadas doses elevadas de colecalciferol (500.000 IU) anualmente (RR=1,08; IC95\%, 1,03-1,14). Na avaliação dos restantes cinco ECA verificou-se uma diferença não significativa no risco de queda (IRR=0,97; IC95\%, 0,79-1,2; $I^{2}=75,8 \%$ ) (Tabela 2). De notar que três ECA recrutaram participantes com elevado risco de queda e cinco ECA envolveram exclusivamente mulheres. Em apenas dois ECA foi adicionado cálcio (500mg/d ou 1000mg/d) à vitamina D (Tabela 3). Numa análise adicional, os autores acrescentaram estudos que recrutaram participantes com níveis insuficientes ou défice de vitamina $D$, inicialmente excluídos, não se verificando uma diferença estatisticamente significativa no número de pessoas que experienciaram quedas (RR=0,88; IC95\%, 0,78-1,00).

Por outro lado, a MA de Cameron ID e colaboradores (2018) ${ }^{12}$ mostrou que em idosos institucionalizados a suplementação com vitamina $\mathrm{D}$ reduz a taxa de quedas ( $\mathrm{RaR}=0,72$; IC95\%, 0,55-0,95; $\left.\mathrm{I}^{2}=62 \%\right)$, mas sem diferença no risco de queda (RR=0,92; IC95\%, 0,76$-1,12 ; I^{2}=42 \%$ ), mesmo quando associada com cálcio. Esta discrepância poderá dever-se às características 
particulares dos indivíduos envolvidos. A média dos níveis séricos basais de vitamina $D$ registados foi baixa ou muito baixa nos cinco ECA incluídos, o que significa que estes resultados se aplicam a indivíduos institucionalizados com baixos níveis de vitamina $\mathrm{D}$.

Wu e colaboradores $(2017)^{13}$ avaliaram o efeito da vitamina D em idosos institucionalizados e não institucionalizados ao longo de dois meses a três anos, sugerindo que a redução do risco de queda depende do tipo da suplementação de vitamina D. Isto é, verificou-se uma redução do risco de queda com a vitamina $\mathrm{D}$ combinada com o cálcio quando comparada com o controlo $\left(\mathrm{OR}=0,87 ; \mathrm{IC95 \% ,} \mathrm{0,80-1,94;} \mathrm{I}^{2}=46 \%\right.$ ). No entanto, não se verificou uma associação entre a suplementação com vitamina $\mathrm{D}_{2}\left(\mathrm{OR}=0,77\right.$; IC95\%, 0,58-1,03; $\left.\mathrm{I}^{2}=79 \%\right)$ e $\mathrm{D}_{3}$ $\left(\mathrm{OR}=1,08 ; \mathrm{IC} 95 \%, 0,98-1,20 ; \mathrm{I}^{2}=42 \%\right)$ e a diminuição das quedas, o que significa que o uso de vitamina $\mathrm{D}$ isoladamente não tem qualquer efeito no risco de quedas. Sabe-se que a vitamina D promove a absorção de cálcio e ajuda a manter as concentrações de cálcio adequadas de forma a manter a mineralização óssea normal.

A partir da análise do Tabela 4 percebe-se que a maioria das MA existentes inclui estudos com toma diária de vitamina $\mathrm{D}$ em doses baixas (400-800 IU). Zheng e colaboradores $(2015)^{14}$ tentaram perceber quais os efeitos da suplementação de elevadas doses e intermitentes de vitamina $\mathrm{D}$ em indivíduos idosos, tendo verificado que esta abordagem não tem benefício na prevenção de fraturas e quedas, podendo até ter efeitos deletérios. Estes resultados indicam que a posologia pode ser mais importante do que a dose total nos resultados finais. Ainda não existe nenhuma explicação para este fenómeno. De destacar que a maioria dos participantes nos estudos incluídos são mulheres idosas. Além disso, a análise agrupada no risco de queda foi influenciada por dois ECA, em que os participantes apresentavam concentrações séricas elevadas de 25(HO)D, o que significa que este risco poderá não se aplicar a pessoas com níveis séricos baixos.

A MA de Bolland e colaboradores (2014) $)^{15}$ demonstrou que, em idosos não institucionalizados, a suplementação de vitamina D com ou sem cálcio não está associada a uma redução do risco de fratura total $(p=0,4)$ e de fratura da anca $(p=0,004)$ igual ou superior a $15 \%$ (limite definido pelos autores como clinicamente relevante). Contudo, os resultados dos estudos que avaliaram o efeito da vitamina $\mathrm{D}$ isoladamente ou combinado com cálcio na fratura da anca devem ser interpretados cautelosamente, tendo em conta as diferentes metodologias utilizadas. De notar, a existência de dois ECA nos quais a vitamina D combinada com o cálcio foi associada a uma redução da fratura da anca em indivíduos institucionalizados. Estes estudos envolveram mulheres com níveis basais baixos de $25(\mathrm{OH}) \mathrm{D}$ e baixa ingestão de cálcio.

A maioria dos estudos envolve idosos sem défice cognitivo e poucos utilizam doentes com esta condição. Assim, Gui e colaboradores (2013) ${ }^{16}$ tentaram perceber quais os efeitos da vitamina $\mathrm{D}$ na prevenção de quedas em idosos com ou sem défices cognitivos, tendo verificado que a suplementação de vitamina D combinada com cálcio reduz o número de quedas em indivíduos não institucionalizados e sem défice cognitivo (OR=0,789; IC95\%, 0,631-0,985; $p=0,036$ ), mas não em indivíduos com défice cognitivo. Contudo, estes resultados não se verificaram quando usada vitamina $D$ isoladamente (OR=1,026; IC95\%, 0,819-1,286; $p=0,036$ ). As limitações deste estudo incluem definições inconsistentes quanto ao défice cognitivo entre os diferentes ensaios.

Gillespie e colaboradores (2012) ${ }^{17}$ demonstraram que em idosos não institucionalizados a suplementação de vitamina $\mathrm{D}$ isolada ou em combinação com o cálcio não reduz a taxa de quedas (RaR=1,00; IC95\%, 0,90$-1,11 ; p<0,00001)$, o risco de queda (RR=0,96; IC95\%, $0,89-1,03 ; p<0,00001)$ ou o risco de fratura (RR=0,94; IC95\%, 0,82-1,09; $p<0,00001)$, mesmo nos estudos que recrutaram indivíduos com elevado risco de fratura. Contudo, ao analisar os quatro ECA que recrutaram apenas indivíduos com níveis séricos basais de 25(HO)D baixos, os autores verificaram uma redução da taxa de quedas (RaR=0,57; IC95\%, 0,37-0,89; $p=0,01) \mathrm{e}$ do risco de queda (RR=0,70; IC95\%, $0,56-0,87 ; p=0,003$ ) associada à suplementação de vitamina $\mathrm{D}$ com ou sem cálcio.

Lai e colaboradores $(2010)^{18}$ mostraram não existir diferença no risco de fratura da anca entre indivíduos que receberam suplementos de vitamina $D_{3}$ ou vitamina $D_{2}$ comparativamente com placebo/controlo ( $\mathrm{RR}=1,13$; IC95\%, 0,98-1,29; $\left.p=0,73 ; \chi^{2}=4,44\right)$, nomeadamente naqueles que receberam elevadas doses de vitamina $D$ 


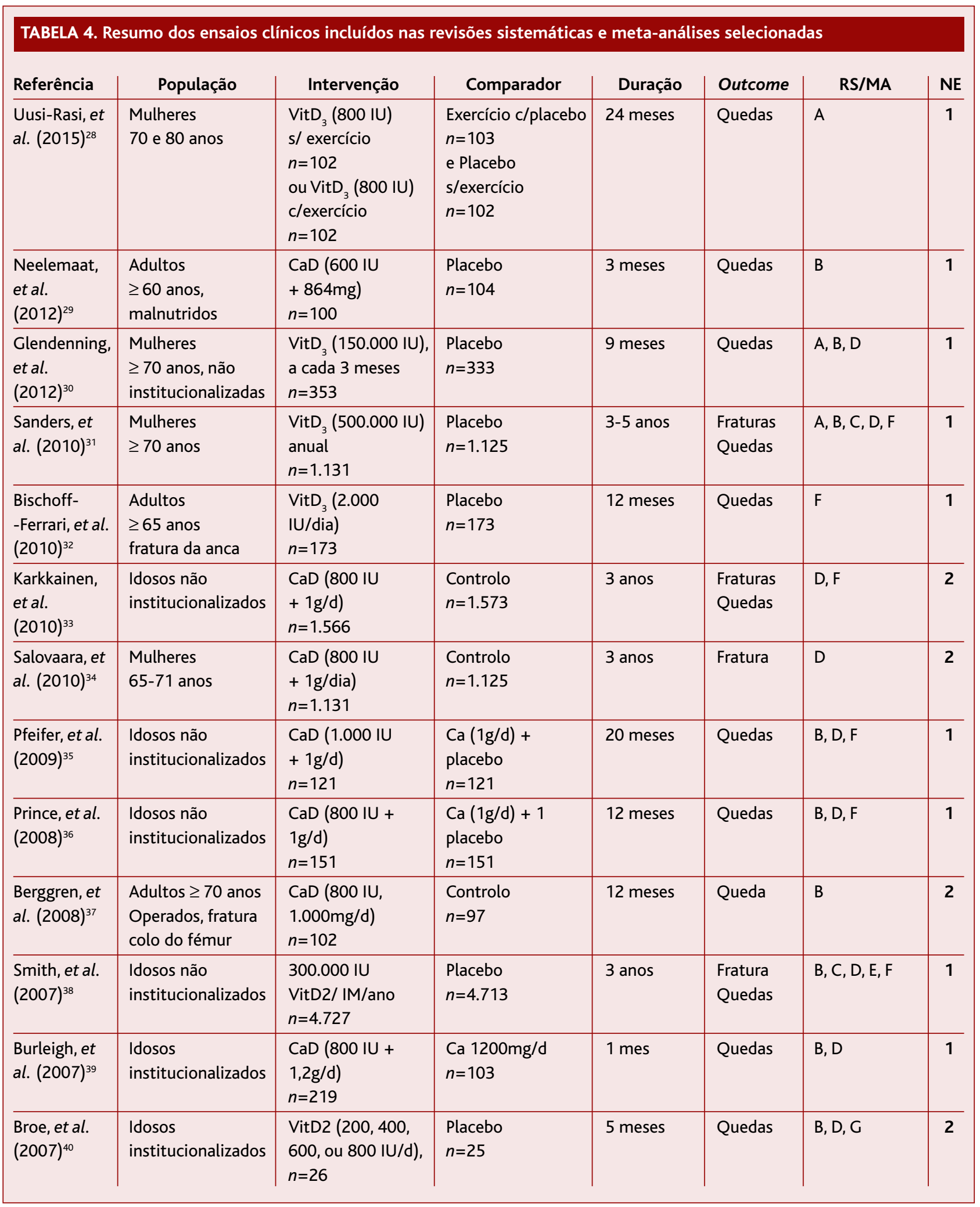




\begin{tabular}{|c|c|c|c|c|c|c|c|}
\hline Referência & População & Intervenção & Comparador & Duração & Outcome & RS/MA & NE \\
\hline $\begin{array}{l}\text { Bischoff, et } \\
\text { al. }(2006)^{41}\end{array}$ & $\begin{array}{l}\text { Mulheres } \geq 65 \\
\text { anos, não } \\
\text { institucionalizadas }\end{array}$ & $\begin{array}{l}\mathrm{CaD}(600 \mathrm{IU}+ \\
400 \mathrm{mg} / \mathrm{d}) \\
n=33\end{array}$ & $\begin{array}{l}\text { Ca } 600 \mathrm{mg} / \mathrm{d} \\
n=31\end{array}$ & 3 meses & $\begin{array}{l}\text { Risco de } \\
\text { queda }\end{array}$ & $B, F, D$ & 1 \\
\hline $\begin{array}{l}\text { Bischoff, et } \\
\text { al. }(2006)^{42}\end{array}$ & $\begin{array}{l}\text { Idosos não } \\
\text { institucionalizados }\end{array}$ & $\begin{array}{l}\mathrm{CaD}(700 \mathrm{IU}+ \\
500 \mathrm{mg} / \mathrm{d}) \\
n=219\end{array}$ & $\begin{array}{l}\text { Placebo } \\
n=226\end{array}$ & 3 anos & Quedas & $A, B, D$ & 1 \\
\hline $\begin{array}{l}\text { Law, et al. } \\
(2006)^{43}\end{array}$ & $\begin{array}{l}\text { Idosos } \\
\text { institucionalizados }\end{array}$ & $\begin{array}{l}\text { VitD2 } \\
(100.000 \mathrm{IU}) \text { a } \\
\text { cada } 3 \text { meses }\end{array}$ & Sem tratamento & 10 meses & $\begin{array}{l}\text { Fratura, } \\
\text { Queda }\end{array}$ & $B, C, E, G$ & 2 \\
\hline $\begin{array}{l}\text { Sato, et al. } \\
(2005)^{44}\end{array}$ & $\begin{array}{l}\text { Mulheres idosas } \\
\text { com hemiplegia } \\
\text { pós-AVC }\end{array}$ & $\begin{array}{l}\text { VitD2 } 1.000 \mathrm{lU} / \mathrm{d} \\
n=48\end{array}$ & $\begin{array}{l}\text { Placebo } \\
n=48\end{array}$ & 24 meses & Queda & B & 2 \\
\hline $\begin{array}{l}\text { Porthouse, et } \\
\text { al. }(2005)^{45}\end{array}$ & $\begin{array}{l}\text { Idosos não } \\
\text { institucionalizados }\end{array}$ & $\begin{array}{l}\mathrm{CaD}(800 \mathrm{IU}+ \\
1 \mathrm{~g} / \mathrm{d}) \\
n=1.321\end{array}$ & $\begin{array}{l}\text { Controlo } \\
n=1.993\end{array}$ & 25 meses & $\begin{array}{l}\text { Fraturas } \\
\text { Quedas }\end{array}$ & $A, D, F$ & 2 \\
\hline $\begin{array}{l}\text { Lyons, et al. } \\
(2007)^{46}\end{array}$ & $\begin{array}{l}\text { Idosos } \\
\text { institucionalizados }\end{array}$ & $\begin{array}{l}\text { VitD2 } \\
\text { ( } 100.000 \mathrm{IU}), \mathrm{a} \\
\text { cada } 4 \text { meses } \\
n=1.715\end{array}$ & $\begin{array}{l}\text { Placebo } \\
n=1.715\end{array}$ & 3 anos & Fratura & $C, E$ & 1 \\
\hline $\begin{array}{l}\text { Larsen, et al. } \\
(2005)^{47}\end{array}$ & $\begin{array}{l}\text { Adultos } \\
\geq 65 \text { anos }\end{array}$ & $\begin{array}{l}\mathrm{CaD}(400 \mathrm{IU}+ \\
1 \mathrm{~g} / \mathrm{d}) \\
n=2.491\end{array}$ & $\begin{array}{l}\text { Controlo } \\
n=2.116\end{array}$ & 42 meses & Quedas & B & 2 \\
\hline $\begin{array}{l}\text { Grant, et al. } \\
(2005)^{48}\end{array}$ & $\begin{array}{l}\text { Idosos não } \\
\text { institucionalizados }\end{array}$ & $\begin{array}{l}\text { VitD3 (800 IU/d), } \\
\text { CaD (800 IU + } \\
\text { 1g/d), } \\
n=2.649\end{array}$ & $\begin{array}{l}\text { Cálcio }(1 \mathrm{~g} / \mathrm{d}), \\
\text { placebo } \\
n=2.643\end{array}$ & 45 meses & $\begin{array}{l}\text { Fraturas } \\
\text { Quedas }\end{array}$ & $B, E, F$ & 2 \\
\hline $\begin{array}{l}\text { Flicker, et al. } \\
(2005)^{49}\end{array}$ & $\begin{array}{l}\text { Idosos } \\
\text { institucionalizados }\end{array}$ & $\begin{array}{l}\mathrm{CaD}(1.000 \mathrm{lU}+ \\
600 \mathrm{mg} / \mathrm{d}) \\
n=313\end{array}$ & $\begin{array}{l}\text { Cácio + placebo } \\
(600 \mathrm{mg} / \mathrm{d}) \\
n=312\end{array}$ & 2 anos & Quedas & $B, D, G$ & 1 \\
\hline $\begin{array}{l}\text { Harwood, et } \\
\text { al. }(2004)^{50}\end{array}$ & $\begin{array}{l}\text { Idosos não } \\
\text { institucionalizados }\end{array}$ & $\begin{array}{l}\text { Vit D3 } \\
(300.000 \mathrm{IU} \text { IM) } \\
\text { CaD }(300.000 \mathrm{IU} \\
+1 \mathrm{~g} / \mathrm{d} \mathrm{IM}) \text { e } \mathrm{CaD} \\
(800 \mathrm{IU}+1 \mathrm{~g} / \mathrm{d} \\
\text { oral) } \\
n=113\end{array}$ & $\begin{array}{l}\text { Controlo } \\
n=37\end{array}$ & 1 ano & Quedas & $B, D, F$ & 2 \\
\hline $\begin{array}{l}\text { Dhesi, et al. } \\
(2004)^{51}\end{array}$ & $\begin{array}{l}\text { Idosos não } \\
\text { institucionalizados } \\
\text { com história de } \\
\text { queda }\end{array}$ & $\begin{array}{l}\text { VitD2 } \\
\text { (600.000 IU/IM) } \\
\text { dose única) } \\
n=70\end{array}$ & $\begin{array}{l}\text { Placebo } \\
n=69\end{array}$ & 6 meses & Queda & $B, C, F, D$ & 1 \\
\hline $\begin{array}{l}\text { Dukas, et al. } \\
(2004)^{52}\end{array}$ & $\begin{array}{l}\text { Idosos não } \\
\text { institucionalizados }\end{array}$ & $\begin{array}{l}\text { VitD3 (40 UI) } \\
n=192\end{array}$ & $\begin{array}{l}\text { Placebo } \\
n=186\end{array}$ & 36 semanas & Quedas & A & 1 \\
\hline
\end{tabular}




\begin{tabular}{|c|c|c|c|c|c|c|c|}
\hline Referência & População & Intervenção & Comparador & Duração & Outcome & RS/MA & NE \\
\hline $\begin{array}{l}\text { Trivedi, et al. } \\
(2003)^{53}\end{array}$ & $\begin{array}{l}\text { Idosos não } \\
\text { institucionalizados }\end{array}$ & $\begin{array}{l}\text { VitD3 (100.000 IU) } \\
\text { cada } 4 \text { meses } \\
n=1.345\end{array}$ & $\begin{array}{l}\text { Placebo } \\
n=1.341\end{array}$ & 60 meses & $\begin{array}{l}\text { Fraturas } \\
\text { Quedas }\end{array}$ & $B, C, D, E, F$ & 1 \\
\hline $\begin{array}{l}\text { Latham, et al. } \\
(2003)^{54}\end{array}$ & Adultos $\geq 65$ anos & $\begin{array}{l}\text { VitD2 (300.000 IU) } \\
\text { oral, dose única + } \\
\text { exercício } \\
n=108\end{array}$ & $\begin{array}{l}\text { Placebo } \\
n=114\end{array}$ & 6 meses & Quedas & $B, C, D, F$ & 1 \\
\hline $\begin{array}{l}\text { Bischoff, et } \\
\text { al. }(2003)^{55}\end{array}$ & $\begin{array}{l}\text { Mulheres } \\
\geq 60 \text { anos } \\
\text { institucionalizadas }\end{array}$ & $\begin{array}{l}\mathrm{CaD}(800 \mathrm{IU}+ \\
1,2 \mathrm{~g}) \\
n=62\end{array}$ & $\begin{array}{l}\text { Ca }(1,2 \mathrm{~g} / \mathrm{d}) \\
n=60\end{array}$ & 3 meses & Quedas & $B, D$ & 1 \\
\hline $\begin{array}{l}\text { Gallagher, et } \\
\text { al. }(2001)^{49}\end{array}$ & $\begin{array}{l}\text { Mulheres idosas } \\
65-77 \text { anos }\end{array}$ & $\begin{array}{l}\text { VitD2 (10 IU, } \\
2 x / d i a) \\
n=123 \\
\text { ou VitD2 (10 IU, } \\
2 x / d i a)+ \text { THS } \\
n=122\end{array}$ & $\begin{array}{l}\text { Estrogénios } \\
0,625 \mathrm{mg} / \mathrm{d} \\
\text { (mulheres sem } \\
\text { útero) ou } \\
\text { estrogénios } \\
0,625 \mathrm{mg} / \mathrm{d}+ \\
\text { acetato de } \\
\text { medroxiprogeste- } \\
\text { rona } 2,5 \mathrm{mg} / \mathrm{d} \\
\text { (mulheres com } \\
\text { útero) } \\
n=121 \\
\text { Ou Placebo } \\
n=123\end{array}$ & 3 anos & Quedas & A & 1 \\
\hline $\begin{array}{l}\text { Chapuy, et al. } \\
(2002)^{56}\end{array}$ & $\begin{array}{l}\text { Idosos } \\
\text { institucionalizados }\end{array}$ & $\begin{array}{l}\mathrm{CaD}(800 \mathrm{IU}+ \\
1,2 \mathrm{~g} / \mathrm{d}) \\
n=389\end{array}$ & $\begin{array}{l}\text { Placebo } \\
n=190\end{array}$ & 24 meses & Quedas & $B, D, G$ & 1 \\
\hline $\begin{array}{l}\text { Pfeifer, et al. } \\
(2000)^{57}\end{array}$ & $\begin{array}{l}\text { Idosos não } \\
\text { institucionalizados }\end{array}$ & $\begin{array}{l}\mathrm{CaD}(800 \mathrm{IU}+ \\
1,2 \mathrm{~g} /) \\
n=74\end{array}$ & $\begin{array}{l}\text { Cálcio }(1,2 \mathrm{~g} / \mathrm{d}) \\
n=74\end{array}$ & 12 meses & Quedas & $B, D, F$ & 1 \\
\hline $\begin{array}{l}\text { Meyer, et al. } \\
(2002)^{58}\end{array}$ & $\begin{array}{l}\text { Idosos } \\
\text { institucionalizados }\end{array}$ & $\begin{array}{l}400 \text { IU VitD3 (oral) } \\
n=569\end{array}$ & $\begin{array}{l}\text { Placebo } \\
n=575\end{array}$ & $\begin{array}{l}2 \text { anos } \\
\text { anca }\end{array}$ & Fratura da & $E$ & 1 \\
\hline $\begin{array}{l}\text { Lips, et al. } \\
(1996)^{59}\end{array}$ & $\begin{array}{l}\text { Idosos } \\
\text { institucionalizados }\end{array}$ & $\begin{array}{l}\text { VitD3 (400 IU) } \\
n=1.291\end{array}$ & $\begin{array}{l}\text { Placebo } \\
n=1.287\end{array}$ & 3,5 anos & $\begin{array}{l}\text { Fratura da } \\
\text { anca }\end{array}$ & $E$ & 1 \\
\hline $\begin{array}{l}\text { Graafmans, et } \\
\text { al. }(1996)^{60}\end{array}$ & $\begin{array}{l}\text { Idosos } \\
\text { institucionalizados }\end{array}$ & $\begin{array}{l}\text { VitD3 }(400 \mathrm{lU} / \mathrm{d}) \\
n=177\end{array}$ & $\begin{array}{l}\text { Placebo } \\
n=177\end{array}$ & 28 semanas & $\begin{array}{l}\text { Fraturas } \\
\text { Quedas }\end{array}$ & $B, D$ & 1 \\
\hline
\end{tabular}

Legenda: A = Guirguis-Blake, et al. (2018); ${ }^{11} \mathrm{~B}=\mathrm{WuH}$, et al. (2017); ${ }^{13} \mathrm{C}=$ Zheng, et al. (2015); ${ }^{14} \mathrm{D}=$ Bolland, et al. (2014); ${ }^{15} \mathrm{E}=$ Lai, et al. (2010); ${ }^{18}$ $\mathrm{F}=$ Gillespie, et al. (2012); ${ }^{17} \mathrm{G}=$ Cameron, et al. (2018); ${ }^{27}$ VitD3 = Colecalciferol; VitD2 = Ergocalciferol/Calcitriol; CaD = Coadministração de Cálcio e Vitamina D.

(800 IU/dia ou mais). Note-se que os diferentes estudos incluem participantes com diferentes níveis basais de 25(HO)D.

\section{Ensaios clínicos aleatorizados controlados não incluídos nas MA e RS}

Foi selecionado um ECA não incluído nas RS e MA 
supracitadas (Tabela 3). Smith e colaboradores (2017) ${ }^{19}$ estudaram o efeito da suplementação de diferentes doses de vitamina $\mathrm{D}_{3}$ nas quedas em idosos com níveis basais séricos de $25(\mathrm{OH}) \mathrm{D}$ inferior a $20 \mathrm{ng} / \mathrm{ml}$. Assim, após 12 meses de tratamento verificou-se uma diminuição da taxa de indivíduos que tiveram pelo menos um episódio de queda com o uso de uma dose média de vitamina $\mathrm{D}_{3}, 1.600,2.400$ e 3.200 IU diária, comparativamente com o placebo $(\mathrm{OR}=3,86$; IC95\%, 1,24 -12,04; $p=0,0024)$, mas que esta diferença não foi observada na dose diária de 400-800 IU. Além disso, a utilização de doses mais elevadas de 4.000 e 4.800 IU diariamente foram associadas a um aumento da taxa de indivíduos que tiveram pelo menos um episódio de queda, comparativamente ao grupo que recebeu doses intermédias (OR=5,6; IC95\%, 2,1-14,8). De referir que a taxa de quedas foi muito maior nos indivíduos com história prévia de queda e $100 \%$ deste grupo teve queda nos dois grupos que receberam elevadas doses de vitamina D. Note-se ainda que, após os 12 meses, se registou uma diminuição da taxa de quedas no grupo que atingiu níveis de $32-41 \mathrm{ng} / \mathrm{ml}$ de $25(\mathrm{OH}) \mathrm{D}$, enquanto aqueles que obtiveram níveis superiores, $40-45 \mathrm{ng} / \mathrm{ml}$ de $25(\mathrm{OH}) \mathrm{D}$, registaram um aumento da taxa de queda.

\section{CONCLUSÃO}

Apesar da maioria das guidelines e NOC ser a favor da suplementação da vitamina $\mathrm{D}$ para a prevenção de quedas e fraturas em idosos, os resultados obtidos através da análise das RS e MA e ECA são conflituosos, pelo que estas poderão ter de ser reconsideradas.

Face às características intrínsecas à condição, é importante fazer a distinção entre idosos institucionalizados e não institucionalizados. Assim, parece não existir um benefício associado à suplementação de vitamina D na prevenção de quedas em idosos não institucionalizados com níveis séricos normais de $25(\mathrm{OH}) \mathrm{D}$ (SORT B). Contudo, o mesmo poderá não se aplicar a indivíduos com níveis basais séricos baixos. Poderá ainda existir uma associação entre a suplementação de vitamina D combinada com cálcio e a redução do risco de queda em idosos não institucionalizados, o que carece de confirmação. Por outro lado, existe a possibilidade de a suplementação de vitamina $\mathrm{D}$, sobretudo combinada com o cálcio, estar indicada em idosos institucionalizados (SORT B). A maioria dos estudos realizados nesta população envolveu indivíduos com níveis basais $25(\mathrm{OH}) \mathrm{D}$ baixos, levantando a questão se estes resultados se aplicarão àqueles com níveis normais. Assim, a ausência de efeito da vitamina D demonstrada em alguns estudos poderá estar relacionada com o facto dos participantes envolvidos nos estudos não terem níveis de 25(OH)D suficientemente baixos de forma a terem algum benefício, pelo que será importante comprovar se os efeitos pretendidos são dependentes dos níveis séricos basais de 25(OH)D. À data não existem estudos que comparem os efeitos do rastreio do défice de vitamina $D$ com a ausência do rastreio. ${ }^{20}$ Os resultados obtidos poderão dever-se ainda aos níveis baixos de vitamina $\mathrm{D}$ utilizados pela maioria dos estudos. Doses intermédias de 1.600, 2.400 e 3.200 IU parecem ser as mais eficazes (Tabela 3 ). No entanto, faltam estudos que confirmem a dose ótima de vitamina D. Por outro lado, parece consensual que doses elevadas de vitamina D administradas de forma intermitente poderão ter efeito no aumento de quedas e fraturas em idosos (SORT B). Alguns autores sugerem a suplementação de vitamina $\mathrm{D}$ associada com o cálcio, apoiando-se na sua ação sinérgica. Contudo, os efeitos parecem ser não dependentes do tipo de vitamina $\mathrm{D}$ que é utilizada.

A análise das RS e MA revela que a maioria dos estudos é de boa qualidade (NE 1), apresentando um tamanho de amostra considerável (Tabela 4). Porém, existe ainda uma elevada heterogeneidade entre os ensaios, pelo que no futuro será importante realizar estudos com metodologias mais uniformizadas, nomeadamente envolvimento similar de homens e mulheres. Será ainda importante fazer a análise individualizada em relação a indivíduos institucionalizados e não institucionalizados. Além disso, ainda não está esclarecido de que forma o estado cognitivo do indivíduo idoso poderá influenciar os resultados.

\section{REFERÊNCIAS BIBLIOGRÁFICAS}

1. World Health Organization. WHO global repont on falls prevention in older age. Geneva:WHO; 2007. ISBN 9789241563536

2. Neo JJ, Kong KH. Prevalence of vitamin D deficiency in elderly patients admitted to an inpatient rehabilitation unit in tropical Singapore. Rehabil Res Pract. 2016;2016:ID9689760.

3. Smith G, Wimalawansa S, Laillou A, Sophonneary P, Un S, Hong R, et al. High prevalence of vitamin D deficiency in Cambodian women: a 
common deficiency in a sunny country. Nutrients. 2016;8(5):290.

4. Grossman DC, Curry SJ, Owens DK, Barry MJ, Caughey AB, Davidson $\mathrm{KW}$, et al. Interventions to prevent falls in community-dwelling older adults: US Preventive Services Task Force Recommendation Statement. JAMA. 2018;319(16):1696-704.

5. Guirguis-Blake JM, Michael YL, Perdue LA, Coppola EL, Beil TL. Interventions to prevent falls in older adults: updated evidence report and systematic review for the US Preventive Services Task Force. JAMA. 2018;319(16):1705-16.

6. Royal Osteoporosis Society. Vitamin D and bone health: a practical clinical guideline for patient management [homepage]. Cesham: ROS; 2018 [updated 2019 Mar 5]. Available from: https://www.guidelines.co.uk/ musculoskeletal-and-joints-/ros-vitamin-d-and-bone-health-guideline/454558.article

7. American Geriatrics Society. American Geriatrics Society Consensus Statement: vitamin $D$ for prevention of falls and their consequences in older adults [Internet]. New York: AGS; 2014. Available from: https://pdfs.semanticscholar.org/a7a1/b6b76f9e45f5d4b5d1f1c0c02b 4e44a5f972.pdf

8. Dawson-Hughes B, Mithal A, Bonjour JP, Boonen S, Burckhardt P, Fuleihan $\mathrm{EH}$, et al. IOF position statement: vitamin $D$ recommendations for older adults. Osteoporos Int. 2010;21(7):1151-4.

9. Direção-Geral da Saúde. Orientação técnica sobre suplementação de cálcio e vitamina $D$ em pessoas idosas: circular informativa $n .{ }^{\circ}$ 13/DSDC/DSQC, de 01/04/2008. Lisboa: DGS; 2008.

10. Holick MF, Binkley NC, Bischoff-Ferrari HA, Gordon CM, Hanley DA, Heaney RP, et al. Evaluation, treatment, and prevention of vitamin D deficiency: an Endocrine Society Clinical Practice Guideline. J Clin Endocrinol Metab. 2011;96(7):1911-30.

11. Guirguis-Blake JM, Michael YL, Perdue LA, Coppola EL, Beil TL, Thompson $\mathrm{JH}$. Interventions to prevent falls in community-dwelling older adults: a systematic review for the U.S. Preventive Services Task Force [Internet]. Rockville:Agency for Healthcare Research and Quality; 2018. Available from: https://www.ncbi.nlm.nih.gov/books/NBK525700/

12. Cameron ID, Dyer SM, Panagoda CE, Murray GR, Hill KD, Cumming RG, et al. Interventions for preventing falls in older people in care facilities and hospitals. Cochrane Database Syst Rev. 2018;9(9):CD005465

13. Wu H, Pang Q. The effect of vitamin D and calcium supplementation on falls in older adults. Orthopade. 2017;46(9):729-36.

14. Zheng YT, Cui QQ, Hong YM, Yao WG. A meta-analysis of high dose, intermittent vitamin D supplementation among older adults. PLoS One. 2015;10(1):e0115850.

15. Bolland MJ, Grey A, Gamble GD, Reid IR. The effect of vitamin D supplementation on skeletal, vascular, or cancer outcomes: a trial sequential meta-analysis. Lancet Diabetes Endocrinol. 2014;2(4):30720.

16. Guo JL, Tsai YY, Liao JY, Tu HM, Huang CM. Interventions to reduce the number of falls among older adults with/without cognitive impairment: an exploratory meta-analysis. Int J Geriatr Psychiatry. 2014;29(7):6619.

17. Gillespie LD, Robertson MC, GillespieWJ, Sherrington C, Gates S, Clemson $L M$, et al. Interventions for preventing falls in older people living in the community. Cochrane Database Syst Rev. 2012;(9):CD007146.

18. Lai JK, Lucas RM, Clements MS, Roddam AW, Banks E. Hip fracture risk in relation to vitamin $D$ supplementation and serum 25 -hydroxyvitamin D levels: a systematic review and meta-analysis of randomised controlled trials and observational studies. BMC Public Health. 2010;10(1): 331.

19. Smith LM, Gallagher JC, Suiter C. Medium doses of daily vitamin D decrease falls and higher doses of daily vitamin D3 increase falls: a randomized clinical trial. J Steroid Biochem Mol Biol. 2017;173:317-22.

20. LeBlanc ES, Zakher B, Daeges M, Pappas M, Chou R. Screening for vitamin D deficiency: a systematic review for the U.S. Preventive Services Task Force. Ann Intern Med. 2015;162(2):109-22.

21. Uusi-Rasi K, Patil R, Karinkanta S, Kannus P, Tokola K, Lamberg-Allardt $C$, et al. Exercise and vitamin $D$ in fall prevention among older women: a randomized controlled trial. JAMA Intern Med. 2015;175(5):70311.

22. Neelemaat F, Lips P, Bosmans JE, Thijs A, Seidell JC, van Bokhorst-de van der Schueren MA. Short-term oral nutritional intervention with protein and vitamin $D$ decreases falls in malnourished older adults. J Am Geriatr Soc. 2012;60(4):691-9.

23. Glendenning P, Zhu K, Inderjeeth C, Howat P, Lewis JR, Prince RL. Effects of three-monthly oral 150,000 IU cholecalciferol supplementation on falls, mobility, and muscle strength in older postmenopausal women: a randomized controlled trial. J Bone Miner Res. 2012;27(1): 170-6.

24. Sanders KM, Stuart AL, Williamson EJ, Simpson JA, Kotowicz MA, Young $D$, et al. Annual high-dose oral vitamin $D$ and falls and fractures in older women: a randomized controlled trial. JAMA. 2010;303(18):181522.

25. Bischoff-Ferrari HA, Dawson-Hughes B, Platz A, Orav EJ, Stähelin HB, Willett WC, et al. Effect of high-dosage cholecalciferol and extended physiotherapy on complications after hip fracture: a randomized controlled trial. Arch Intern Med. 2010;170(9):813-20.

26. Kärkkäinen MK, Tuppurainen M, Salovaara K, Sandini L, Rikkonen T, Sirola J, et al. Does daily vitamin D $800 \mathrm{IU}$ and calcium $1000 \mathrm{mg}$ supplementation decrease the risk of falling in ambulatory women aged 6571 years? A 3-year randomized population-based trial (OSTPRE-FPS). Maturitas. 2010;65(4):359-65.

27. Salovaara K, Tuppurainen M, Kärkkäinen M, Rikkonen T, Sandini L, Sirola J, et al. Effect of vitamin D3 and calcium on fracture risk in 65- to 71-year-old women: a population-based 3-year randomized, controlled trial-the OSTPRE-FPS. J Bone Miner Res. 2010;25(7):1487-95.

28. Pfeifer M, Begerow B, Minne HW, Suppan K, Fahrleitner-Pammer A, Dobnig $\mathrm{H}$. Effects of a long-term vitamin $\mathrm{D}$ and calcium supplementation on falls and parameters of muscle function in community-dwelling older individuals. Osteoporos Int. 2009;20(2):315-22.

29. Prince RL, Austin N, Devine A, Dick IM, Bruce D, Zhu K. Effects of ergocalciferol added to calcium on the risk of falls in elderly high-risk women. Arch Intern Med. 2008;168(1):103-8.

30. Berggren M, Stenvall M, Olofsson B, Gustafson Y. Evaluation of a fallprevention program in older people after femoral neck fracture: a oneyear follow-up. Osteoporos Int. 2008;19(6):801-9.

31. Smith $H$, Anderson F, Raphael H, Maslin P, Crozier S, Cooper C. Effect of annual intramuscular vitamin $D$ on fracture risk in elderly men and women a population-based, randomized, double-blind, placebo-controlled trial. Rheumatology. 2007;46(12):1852-7. 
32. Burleigh E, McColl J, Potter J. Does vitamin D stop inpatients falling? A randomised controlled trial. Age Ageing. 2007;36(5):507-13.

33. Broe KE, Chen TC, Weinberg J, Bischoff-Ferrari HA, Holick MF, Kiel DP. A higher dose of vitamin $D$ reduces the risk of falls in nursing home residents: a randomized, multiple-dose study. J Am Geriatr Soc. 2007;55(2):234-9.

34. Bischoff-Ferrari HA, Conzelmann M, Stähelin HB, DickW, Carpenter MG Adkin $A L$, et al. Is fall prevention by vitamin $D$ mediated by a change in postural or dynamic balance? Osteoporos Int. 2006;17(5):656-63.

35. Bischoff-Ferrari HA, Orav EJ, Dawson-Hughes B. Effect of cholecalciferol plus calcium on falling in ambulatory older men and women: a 3-year randomized controlled trial. Arch Intern Med. 2006;166(4):42430.

36. Law M, Withers $\mathrm{H}$, Morris J, Anderson F.Vitamin D supplementation and the prevention of fractures and falls: results of a randomised trial in elderly people in residential accommodation. Age Ageing. 2006;35(5): 482-6.

37. Sato Y, Iwamoto J, Kanoko T, Satoh K. Low-dose vitamin D prevents muscular atrophy and reduces falls and hip fractures in women after stroke: a randomized controlled trial. Cerebrovasc Dis. 2005;20(3):187-92.

38. Porthouse J, Cockayne S, King C, Saxon L, Steele E, Aspray T, et al. Randomised controlled trial of calcium and supplementation with cholecalciferol (vitamin D3) for prevention of fractures in primary care. BMJ. 2005;330(7498):1003.

39. Lyons RA, Johansen A, Brophy S, Newcombe RG, Phillips CJ, Lervy B, et al. Preventing fractures among older people living in institutional care: a pragmatic randomised double blind placebo controlled trial of vitamin D supplementation. Osteoporos Int. 2007;18(6):811-8-

40. Larsen ER, Mosekilde L, Foldspang A. Vitamin D and calcium supplementation prevents severe falls in elderly community-dwelling women: a pragmatic population-based 3-year intervention study. Aging Clin Exp Res. 2005; 17:125-32.

41. Grant AM, Avenell A, Campbell MK, McDonald AM, MacLennan GS, McPherson GC, et al. Oral vitamin D3 and calcium for secondary prevention of low-trauma fracture (randomised evaluation of calcium or vitamin $D, R E C O R D)$ : a randomised placebo controlled trial. Lancet. 2005;365(9471):1621-8.

42. Flicker L, Maclnnis RJ, Stein MS, Scherer SC, Mead KE, Nowson CA, et al. Should older people in residential care receive vitamin $D$ to prevent falls? Results of a randomized trial. J Am Geriatr Soc. 2005;53(11):18818.

43. Harwood RH, Sahota O, Gaynor K, Masud T, Hosking DJ, Nottingham Neck of Femur (NONOF) Study. A randomised, controlled comparison of different calcium and vitamin $\mathrm{D}$ supplementation regimens in elderly women after hip fracture: the Nottingham Neck of Femur (NONOF) Study. Age Ageing. 2004;33(1):45-51.

44. Dhesi JK, Jackson SH, Bearne LM, Moniz C, Hurley MV, Swift CG, et al. Vitamin D supplementation improves neuromuscular function in older people who fall. Age Ageing. 2004;33(6):589-95.

45. Dukas L, Bischoff HA, Lindpaintner LS, Schacht E, Birkner-Binder D,
Damm TN, et al. Alfacalcidol reduces the number of fallers in a community-dwelling elderly population with a minimum calcium intake of more than 500 mg daily. J Am Geriatr Soc. 2004;52(2):230-6.

46. Trivedi DP, Doll R, Khaw KT. Effect of four monthly oral vitamin D3 (cholecalciferol) supplementation on fractures and mortality in men and women living in the community: randomised double blind controlled trial. BMJ. 2003;326(7387):469.

47. Latham NK, Anderson CS, Lee A, Bennett DA, Moseley A, Cameron ID, et al. A randomized, controlled trial of quadriceps resistance exercise and vitamin D in frail older people: the Frailty Interventions Trial in Elderly Subjects (FITNESS). J Am Geriatr Soc. 2003;51(3):291-9.

48. Bischoff HA, Stähelin HB, Dick W, Akos R, Knecht M, Salis C, et al. Effects of vitamin $D$ and calcium supplementation on falls: a randomized controlled trial. J Bone Miner Res. 2003;18(2):343-51.

49. Gallagher JC, Fowler SE, Detter JR, Sherman SS. Combination treatment with estrogen and calcitriol in the prevention of age-related bone loss. J Clin Endocrinol Metab. 2001;86(8):3618-28.

50. Chapuy MC, Pamphile R, Paris E, Kempf C, Schlichting M, Arnaud S, et al. Combined calcium and vitamin D3 supplementation in elderly women: confirmation of reversal of secondary hyperparathyroidism and hip fracture risk: the Decalyos II study. Osteoporos Int. 2002;13(3):257-64.

51. Pfeifer M, Begerow B, Minne HW, Abrams C, Nachtigall D, Hansen C. Effects of a short-term vitamin $D$ and calcium supplementation on body sway and secondary hyperparathyroidism in elderly women. J Bone Miner Res. 2000;15(6):1113-8.

52. Meyer HE, Smedshaug GB, Kvaavik E, Falch JA, Tverdal A, Pedersen JI. Can vitamin D supplementation reduce the risk of fracture in the elderly? A randomized controlled trial. J Bone Miner Res. 2002;17(4):70915.

53. Lips P, GraafmansWC, Ooms ME, Bezemer PD, Bouter LM. Vitamin D supplementation and fracture incidence in elderly persons: a randomized, placebo-controlled clinical trial. Ann Intern Med. 1996;124(4):400-6.

54. Graafmans WC, Ooms ME, Hofstee HM, Bezemer PD, Bouter LM, Lips P. Falls in the elderly: a prospective study of risk factors and risk profiles. Am J Epidemiology. 1996;143(11):1129-36.

55. Cameron ID, Gillespie LD, Robertson MC, Murray GR, Hill KD, Cumming $\mathrm{RG}$, et al. Interventions for preventing falls in older people in care facilities and hospitals. Cochrane Database Syst Rev. 2012;12:CD005465.

\section{CONFLITO DE INTERESSES}

Os autores declaram não ter quaisquer conflitos de interesse.

\section{ENDEREÇO PARA CORRESPONDÊNCIA}

Anabela Barreto Silva

E-mail: anabelafbsilva@gmail.com

https://orcid.org/0000-0003-3788-2249

Recebido em 18-19-2019

Aceite para publicação em 31-01-2020 


\section{ABSTRACT \\ SUPPLEMENTATION OF VITAMIN D IN PREVENTION OF FALLS AND FRACTURES IN ELDERLY:AN EVIDENCE-BASED REVIEW}

Introduction: Falls and fractures are an important cause of hospitalization and mortality in elderly individuals, contributing significantly to their loss of autonomy. The role of vitamin D in improving musculoskeletal disorders in elderly patients in the prevention of falls and fractures is still controversial.

Aim: To determine the evidence of the effect of vitamin D supplementation on the prevention of falls and fractures in the elderly.

Data source: MEDLINE, The Cochrane Library, NGC, NICE, DARE, TRIP Database, USPSTF, AGS, and DGS.

Methods: Research of studies published in the last 10 years (from February 1, 2009, to February 28, 2019) written in Portuguese and English using the terms MeSH 'Vitamin D', 'Accidental Falls', and 'Fractures, Bone'. Evaluation of the quality of the studies and strength of recommendation was used in the Strength of Recommendation Taxonomy scale of the American Family Physician (SORT).

Results: From the initial study, 514 articles were selected and 15 were selected because they met the defined inclusion criteria: six guidelines, eight systematic reviews (RS), and meta-analyses (MA) and one randomized clinical trial (RCT). Most guidelines and NOC advocate vitamin D supplementation in the prevention of falls and fractures in the elderly. However, the RS and MA analyzes show discordant results in demonstrating that vitamin D supplementation has no effect on falls and fractures in the elderly, particularly non-institutionalized. The effects may be greater in individuals with low baseline vitamin $D$ levels. Conclusions: There is no benefit of vitamin supplementation in the prevention of falls and fractures (SORT B) in non-institutionalized elderly people without a deficit of $25(\mathrm{OH}) \mathrm{D}$. Institutionalized elderly with a deficit of $25(\mathrm{OH}) \mathrm{D}$ should be supplemented with vitamin D alone or combined with calcium (SORT B). Supplementation of high doses of vitamin D and intermittently may have deleterious effects (SORT B).

Keywords: Vitamin D; Falls; Fractures; Elderly. 